\title{
DETERMINATION OF 2D IMPLANTED ION DISTRIBUTIONS USING INVERSE RADON TRANSFORM METHODS
}

\author{
Eddie VAN SCHIE and Jan MIDDELHOEK \\ University of Twente, P.O.B. 217, 7500 AE Enschede, The Netherlands
}

Peer C. ZALM

Philips Research Laboratories, P.O.B. 80000,5600 JA Eindhoven, The Netherlands

Received 25 October 1988 and in revised form 20 January 1989

Two methods are presented for the experimental determination of $2 \mathrm{D}$ implanted ion distribution resulting from implantations with a line source into amorphous targets. It is shown that the relation between the $2 \mathrm{D}$ distribution and the depth profiles resulting from tilted angle implantations is described by the Radon transformation. The inverse transformation has been applied to accurately measured depth profiles. The first method uses a digitization of the $2 \mathrm{D}$ distribution and the second method uses a parameterized function for the 2D distribution. The methods are tested for a $400 \mathrm{keV}$ boron implantation in an amorphous layer of silicon. The experimental obtained 2D distributions are compared with a TRIM Monte Carlo simulation. A good agreement between experiment and simulation is observed.

\section{Introduction}

When an ion penetrates a substrate it will lose its energy through collisions with substrate atoms and interactions with the substrate electrons. Finally the ion comes to rest. The depth probability distribution of the ions can be described with a distribution function. Originally a Gaussian distribution function was used, which only needs two moments. These are the mean projected range and the projected range straggle (standard deviation). Today it is well established that for a realistic description of depth probability distributions four moments must be taken into account. For this purpose the Pearson IV distribution is commonly used [5].

One of the first models including lateral spread was presented by Furukawa in 1972 [1]. It is based on the statistical distribution function $f(y, x)$ of a line source, with $y$ the vertical coordinate (perpendicular to the surface) and $x$ the lateral coordinate. Furukawa used a 2D Gaussian function for $f(y, x)$, which may be written as:

$f(y, x)=\operatorname{gauss}(y) \operatorname{gauss}(x)$.

The moments of this $2 \mathrm{D}$ distribution function may be obtained either by theory [1] or by experiment [2-4].

\footnotetext{
* This work was supported by the National Innovative Research Program under IOP IC-TEL 45.012.
}

The methods for experimental determination of the moments are generally based on implantations in tilted targets. The projected range straggle of the $1 \mathrm{D}$ profiles of the tilted implants are related to the standard deviations in the 2D distribution function.

Results of Monte Carlo simulations deviate very much from the 2D Gaussian distribution function [5]. A more general 2D distribution function may be written as:

$f(y, x)=f_{\text {dep }}(y) f_{\text {lat }}(x, m(y))$,

where $f_{\text {lat }}(y, x)$ is a lateral distribution function with moments $m$ that depend on the depth $y . f_{\text {dep }}(y)$ is a proper depth distribution function.

In this paper a novel method is introduced to experimentally determine the $2 \mathrm{D}$ distribution function $f(y, x)$. The method is based on several tilted beam implants. It will be shown that the relation between $f(y, x)$ and depth profiles of tilted implants is described by the Radon transformation. This transformation occurs when measured data have the form of line integrals of the spatial distribution of a physical property [6]. Calculations of the inverse transformation gives the desired 2D distribution function.

In section 2 the relation between the $2 \mathrm{D}$ distribution function and depth profiles of tilted beam implants will be derived. In section 3 two methods to perform the inverse transformation will be discussed. In section 4 a Monte Carlo simulation of a $2 \mathrm{D}$ distribution function is discussed, which is used as a test case. In section 5 the 
experimental setup is discussed. In section 6 experimental results of a $400 \mathrm{keV}$ boron implant are presented. Finally in section 7 the conclusions are drawn.

\section{The relation between the $2 D$ distribution function and $1 \mathrm{D}$ profiles}

A line source results in a 2 dimensional distribution $C_{2 \mathrm{D}}(y, x)$, where $y$ is the depth in the substrate and $x$ is the lateral distance from the beam. See fig. 1, where contours are drawn of $C_{2 \mathrm{D}}(y, x)$ for a beam thickness $\mathrm{d} \xi \rightarrow 0$. This 2D distribution can be used to construct depth profiles resulting from tilted implants. These depth profiles can be measured with analysis methods such as SIMS. In this section the relation between the 2D distribution and the measurable depth profiles will be derived. First a proper definition of a $2 \mathrm{D}$ distribution function $C_{2 \mathrm{D}}(y, x)$ is needed.

In order to define $C_{2 \mathrm{D}}(y, x)$, the ion distribution will be calculated of an implantation with a small beam width $d \xi$. The number of ions per unit length, passing the surface is:

$N_{\square} \mathrm{d} \xi$,

where $N_{\square}$ is the implantation dose in ions $/ \mathrm{cm}^{3}$ and $\mathrm{d} \xi$ is the beam width in $\mathrm{cm}$. The number of ions per unit length $N_{y, x}$, ending up in a square at position $y, x$ is then given by:

$N_{y, x}=N_{\square} \mathrm{d} \xi C_{2 D}(y, x) \mathrm{d} y \mathrm{~d} x$,

where: $\mathrm{d} y, \mathrm{~d} x$ are the sides in $\mathrm{cm}$ and $C_{2 \mathrm{D}}(y, x)$ is a probability distribution function in $\mathrm{cm}^{-2}$.

The implantation into a tilted substrate can now be calculated as a summation over shifted responses as shown in fig. 2a. Mathematically this can be formulated as a line integral as shown in fig. $2 b$. The number of ions per unit length $N_{\mathrm{d}}$ at a depth $d$, ending up in a square with sides $\mathrm{d} x$ and $\mathrm{d} y$ is then given by:

$$
\begin{aligned}
N_{\mathrm{d}}= & N_{\square} \cos \theta \mathrm{d} x \mathrm{~d} y \\
& \times \int_{-\infty}^{\infty} C_{2 \mathrm{D}}(d / \cos \theta-\xi \sin \theta, \xi \cos \theta) \mathrm{d} \xi,
\end{aligned}
$$

a

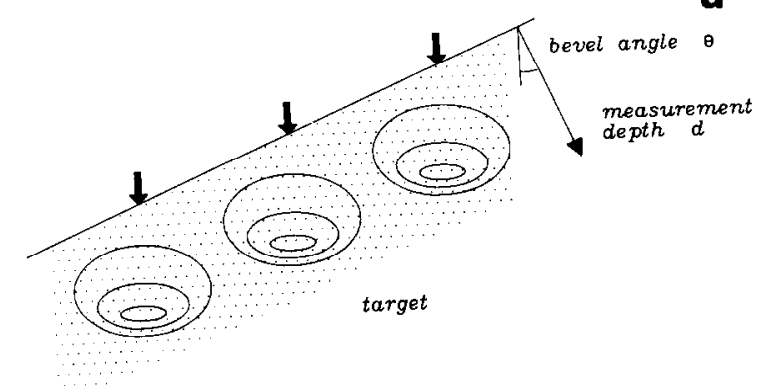

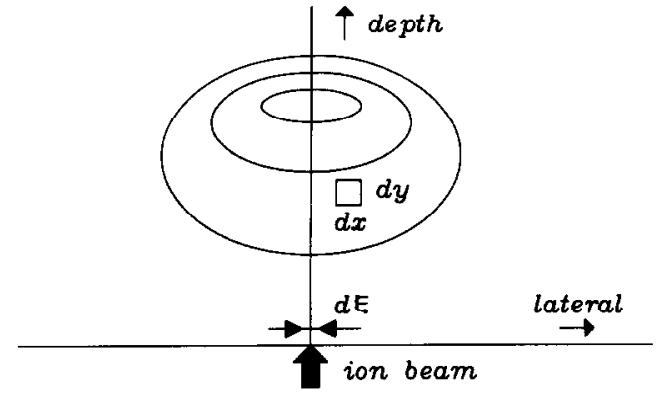

Fig. 1. Contours of 2D distribution resulting from a Line Source with a beam width $d \xi \rightarrow 0$. The ions enter the target at the position indicated by the arrow.

where $\theta$ is the tilt angle and $N_{\square} \cos \theta$ is the effective dose. To obtain a measurable quantity, $N_{\mathrm{d}}$ has to be divided by the square size. Then the concentration $C_{1 \mathrm{D}}$ in ions $/ \mathrm{cm}^{3}$ as a function of the measurement depth $d$ is obtained.

$$
\begin{aligned}
C_{1 \mathrm{D}}(d)= & N_{\square} \cos \theta \\
& \times \int_{-\infty}^{\infty} C_{2 \mathrm{D}}(d / \cos \theta-\xi \sin \theta, \xi \cos \theta) \mathrm{d} \xi .
\end{aligned}
$$

If this transformation is normalized with respect to the effective dose, a pure line integral is obtained.

$p(d)=\int_{-\infty}^{\infty} C_{2 \mathrm{D}}(d / \cos \theta-\xi \sin \theta, \xi \cos \theta) \mathrm{d} \xi$.

This integral is known as the projection integral or Radon transformation [6] and occurs in many other situations as for instance Computerized Tomography (CT). In accordance with the literature concerning projections and their reconstructions, the measurable profiles $p(d)$ will be called projections.

Some limitations on the availability of measured projections exist. First, not a complete range of angles can be obtained. If the tilt angle is near $90^{\circ}$ the back scattering of implanted ions will be very significant, especially if the mass of the impinging ion is lower then the mass of the target atom. This effect causes the line

Fig. 2. a) Shifted Line Source Responses in a bevelled target. b) The resulting 1D depth profile is obtained with line integrals. 
integral to be invalid near the surface. Second for every angle unequal to $0^{\circ}$ a part of $C_{2 \mathrm{D}}$ falls outside the integration area, i.e. the profiles are truncated by the surface. The shaded area in fig. $2 b$ illustrates this part for a certain angle. These limitations constrain the choice of available reconstruction algorithms and limits the application of the technique to cases where the implantation energy is high. The reconstruction methodology is introduced in the next section.

\section{Introduction to reconstruction techniques}

In general three methods can be distinguished for image reconstruction from projections: a) Transform methods, b) algebraic reconstruction techniques (ART) and c) nonlinear optimization techniques (NOT). Transform methods are based on Fourier transformations of the projection integral. However this method is not suitable for the purpose of 2D profiling for two reasons [6]. First an equidistant sampling of data is needed over the complete ranges both in depth and angle. The implantation experiments cannot directly fulfill these demands. Second the $2 \mathrm{D}$ profile has a very steep flank (high contrast image). This will cause alliasing and overshoot problems. Because of these problems the transform methods are not considered here. In this section only the ART and NOT methods are discussed.

\subsection{Algebraic reconstruction techniques (ART)}

\subsubsection{General}

In ref. [7] a detailed review of algebraic reconstruction techniques is presented. To give some idea of the basic principal, this subsection summarizes the application of the ART approach to the reconstruction problem with emphasis on our application. In the next subsection refinements to this basic approach will be discussed. The fundamental model in the ART approach to the image reconstruction problem is formulated in the following way: A Cartesian grid of square picture elements, called pixels, is introduced into the region of interest so that it covers the whole picture which has to be reconstructed. The pixels are numbered in some manner, say from 1 (top left corner pixel) to $n$ (bottom right corner pixel), see fig. 3 . For the $j$ th pixel a value $c_{j}$ is assumed. On this grid the $2 \mathrm{D}$ image is represented with a set of hasis function $\left\{b_{j}(y, x)\right\}_{j=1}^{n}$, The digitization of the 2D image is then given by:

$\hat{C}_{2 \mathrm{D}}(y, x)=\sum_{j=1}^{n} c_{j} b_{j}(y, x)$.

Let $\left(\mathbb{F}_{i}\right)_{i=1}^{m}$ be a set of linear and continuous functionals which assigns to any image $C_{2 \mathrm{D}}(y, x)$ a real number $\mathbb{F}_{i} C$. In our case, $\mathbb{F}_{i} C$ is the line integral (7) of $C_{2 \mathrm{D}}(y, x)$

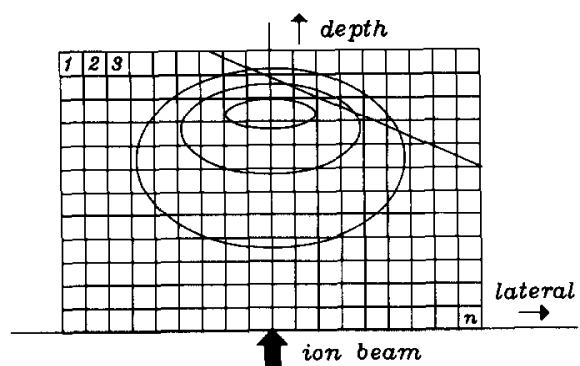

Fig. 3. Discretization of the $2 \mathrm{D}$ distribution to obtain a numerical line integration.

at a certain depth with a certain angle. Now if $p_{i}$ is the measured value for $\mathbb{F}_{i} C$ we may write

$p_{i} \cong \mathbb{F}_{i} C \cong \mathbb{F}_{i} \hat{C}=\sum_{j=1}^{n} c_{j} \mathbb{F}_{i} b_{j}(y, x)=\sum_{j=1}^{n} c_{j} a_{i j}$,

where $a_{i j}=\mathbb{F}_{i} b_{j}(y, x)$. For the case that the basis function $b_{j}$ is 1 if it belongs to the $j$ th element and 0 elsewhere, the coefficient $a_{i j}$ is the length of intersection of the $i$ th line integral with the $j$ th pixel. In matrix notation (9) may be written as

$p \cong \mathbf{A c}$.

where

$\boldsymbol{p}^{\prime}$ is the measurement vector, with dimension $m$ ( $m$ line integrals).

$c \quad$ is the image vector, with the dimension $n$ ( $n$ pixels).

A is the projection matrix, with dimension $m \times n$

The reconstruction problem is now defined as finding an image vector $c$ which reproduces the measurements $p$. The system of equations obtained is sometimes under determined due to a lack of information, often it is greatly over determined in which case it is most probably inconsistent (i.e. there does not exist a solution in the ordinary sense $\boldsymbol{c}=\mathbf{A}^{-1} \cdot \boldsymbol{p}$ ). Moreover, we might have reason to believe that the exact algebraic solution of the system, even if it would exist and we could compute it accurately, is no more desirable in terms of the reconstruction problem than some other, differently defined, "solution". Such a belief may stem from evidence about measurement inaccuracy or noise corruption of data and the fact that the original problem has undergone discretization. The solution techniques which are developed in view of this special features of the system at hand are classified as row action methods. These are iterative solution procedures.

An iteration process starts with an initial approximation $c^{0}$ to the image vector $c$. In an iterative step, the current iterate $c^{k}$ is corrected to a new iterate $c^{k+1}$ by taking into account only a single line integral, say the $i$ th, and changing only the image values of the pixels which intersect this line integral. The discrepancy be- 
tween the measurement $y_{i}$ and the pseudo-projection data

$$
\sum_{j=1}^{n} a_{i j} c_{j}^{k}
$$

obtaincd from the current image $c^{k}$ is redistributed among the pixels along the $i$ th line integral proportionally to their weights $a_{i j}$ in the whole line integral, In this way, the pixel values along the $i$ th line integral are corrected to conform with the $i$ th measurement without changing the rest of the image. Denoting the vector $a_{i}$ as the $i$ th row of the matrix A, a reconstruction process can be described by the following algorithm, ALG1:

\section{Initialization}

$c^{0}=$ start estimation

Typical step

$c^{k+1}=c^{k}+\lambda^{k} \cdot \delta^{i}$

with

$\delta^{i}=0$,

$\delta^{i}=\frac{p_{i}-\left\langle a_{i}, c^{k}\right\rangle}{\left\|a_{i}\right\|^{2}} a_{i}, \quad$ if $\left|p_{i}-\left\langle a_{i}, c^{k}\right\rangle\right| \geq \epsilon_{i}$,

where

$i \quad=$ the index indicating the $i$ th line integral (the line integrals are chosen cyclically, $i=i^{k}=$ $k(\bmod m)+1)$;

$k \quad=$ the index indicating the $k$ th iteration;

$\delta^{i} \quad=$ the correction vector;

$\lambda^{k} \quad=$ a relaxation parameter;

$\epsilon^{i} \quad=$ is a feasibility parameter for the $i$ th projection;

$a^{i} \quad=$ the $i$ th row of the matrix A;

$c^{k} \quad=$ the $k$ th image vector;

$\left\langle a_{i}, c^{k}\right\rangle=$ the inner product of $a^{i}$ and $c^{k}$ (pseudo projection);

$\left\|a_{i}\right\|=$ the Euclidean norm of $a^{i}\left(\left\langle a_{i}, a_{i}\right\rangle\right)$.

Little is known about the mathematical properties of the iterates such as: 1) does the sequence converge, 2) what is the rate of convergence, 3) how do the iterates behave when the data is not consistent. Especially the latter is interesting. In ref. [7] it is stated that the use of relaxation parameters is extremely important for such cases. However no general recipe exists for how such cases should be handled, and the iteration process is stopped when the user of the algorithm sees the image has certain properties.

The application of this algorithm to the reconstruction problem arising from ion implantation does not give satisfactory results. The function which has to be reconstructed is a rapidly varying quantity with values ranging over 3 decades. On a linear scale this means that the function has very steep edges. For more stan- dard reconstruction problems the position of the edge is more important than the form of the edge. For ion implantation however the form of the edge is the information which is most important. In the next subsection the adaptation of the basic algorithm ALG1 is discussed.

\subsubsection{The reconstruction technique for ion implantation}

Because of the rapidly varying reconstruction object the standard approach failed to give accurate results. In the final reconstruction algorithm two adaptations to the standard approach were carried out. First the data was transformed to the logarithmic domain and second a filtering step was introduced.

The most straightforward thing to do was to take the logarithm of the image vector $c$ and to choose another basis function to obtain the digitization of the 2D image. The consequence of this, however, is that the simple matrix equation (10) is not appropriate anymore. The calculation of the pseudo projections and the calculation of the corrections have to be adapted.

The pseudo projections are obtained by using the trapezium rule for the numerical calculation of the line integral (7). The values of $\hat{C}_{2 \mathrm{D}}$ at arbitrary positions inside a pixel are obtained by a bilinear interpolation of the 4 nearest pixel values. Because the logarithmic value is interpolated, the interpolation error is small.

The corrections which must be carried out after comparison of the pseudo projection with the measurement are not straightforward anymore. The following correction is used for all the pixels $j$ which where used to calculate the line integral of a pseudo projection $i$.

Algorithm for ion implantation, ALG2:

\section{Initialization}

$\left[\log \left(c_{j}\right)\right]^{0}=$ start estimation.

Typical step

$\left[\log \left(c_{j}\right)\right]^{k+1}=\left[\log \left(c_{j}\right)\right]^{k}+\lambda \log \left(\delta^{i}\right)$,

with

$$
\begin{array}{ll}
\delta^{i}=1, & \text { if }\left|1-p_{i} / p_{i}^{*}\right|<\epsilon_{i}, \\
\delta^{i}=p_{i} / p_{i}^{*}, & \text { if }\left|1-p_{i} / p_{i}^{*}\right| \geq \epsilon_{i}, \\
\text { where } &
\end{array}
$$

$i=$ the index indicating the $i$ th projection (the projections are chosen cyclically, $i=i^{k}=k(\bmod m)+$ $1)$;

$k=$ the index indicating the $k$ th iteration;

$j=$ the index of the $j$ th pixel used in the calculation of the $i$ th projection;

$\delta^{i}=$ the correction parameter;

$p_{i}^{*}=$ the pseudo projection obtained by numerical integration;

$\lambda=\mathbf{a}$ relaxation parameter;

$\epsilon^{i}=$ is a feasibility parameter for the $i$ th projection;

$c_{j}^{k}=$ the $k$ th $j$ th pixel value. 
This type of correction is equivalent to a relative correction as given by

$c_{j}^{k+1} / c_{j}^{k}=\left(P_{i} / P_{i}^{*}\right)^{\lambda}$.

If a pseudo projection is too large, a corresponding pixel value has to decrease. The relaxation parameter $\lambda$ can increase or decrease the correction. If $\lambda=1$ the pseudo projection, which is calculated with the updated pixel values, will match the measured projection.

Furthermore a filter has been introduced to speed up convergence and to fill pixels which are not covered by
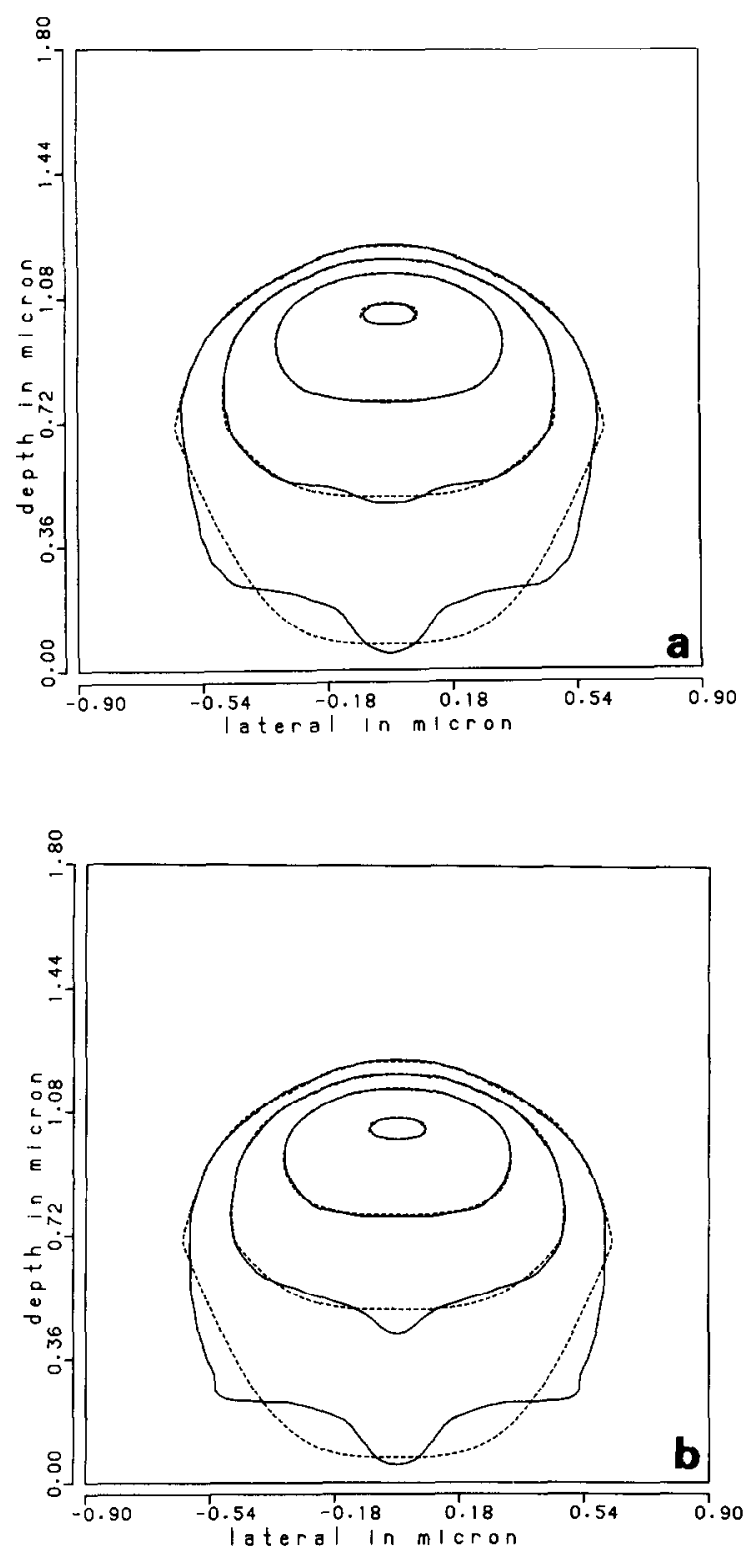

any of the line integrals. The filter contains two parts. The first part is a smoothing operation in two dimensions. It takes the logarithmic average of a block of 9 pixels to replace the middle pixel value. The middle pixel is weighted with 4 , the east, south, west and north pixcls are wcighted with 2 and the remaining pixels are weighted with 1 . So the high frequency components are removed from the distribution. Care must be taken however with the distribution edges because a sort of diffusion occurs. This effect is governed by the number of pixels used to represent the imagc. Furthermore any
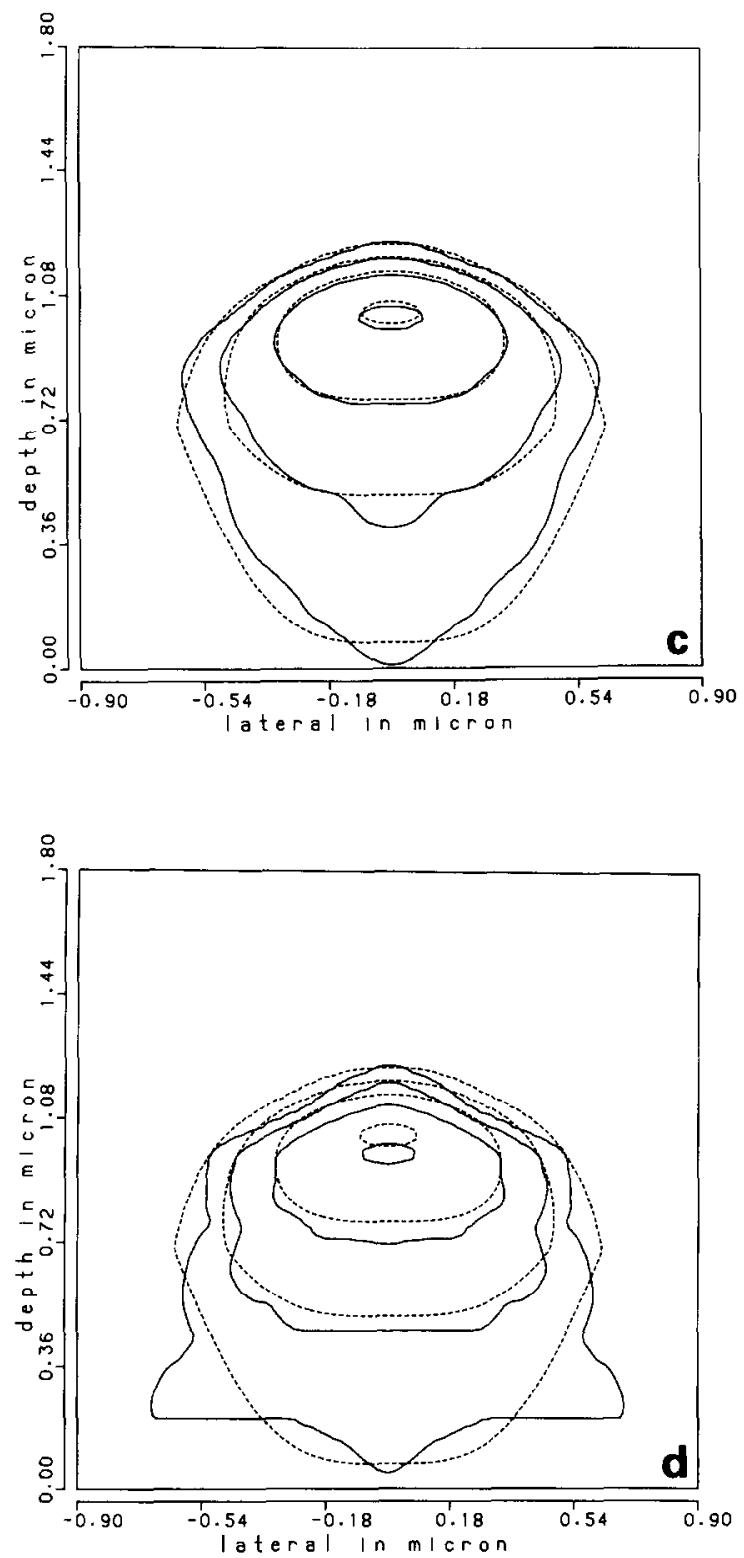

Fig. 4. Reconstructions with ART using simulated profiles. Shown are the contours at $0.9,0.09,0.009$, and 0.0009 below the top. The dotted lines represent the original. a) Result with exact data. b) Result with errors in the dose for different angles. c) Result with errors in the depth scale. d) Result with shifted profiles. 

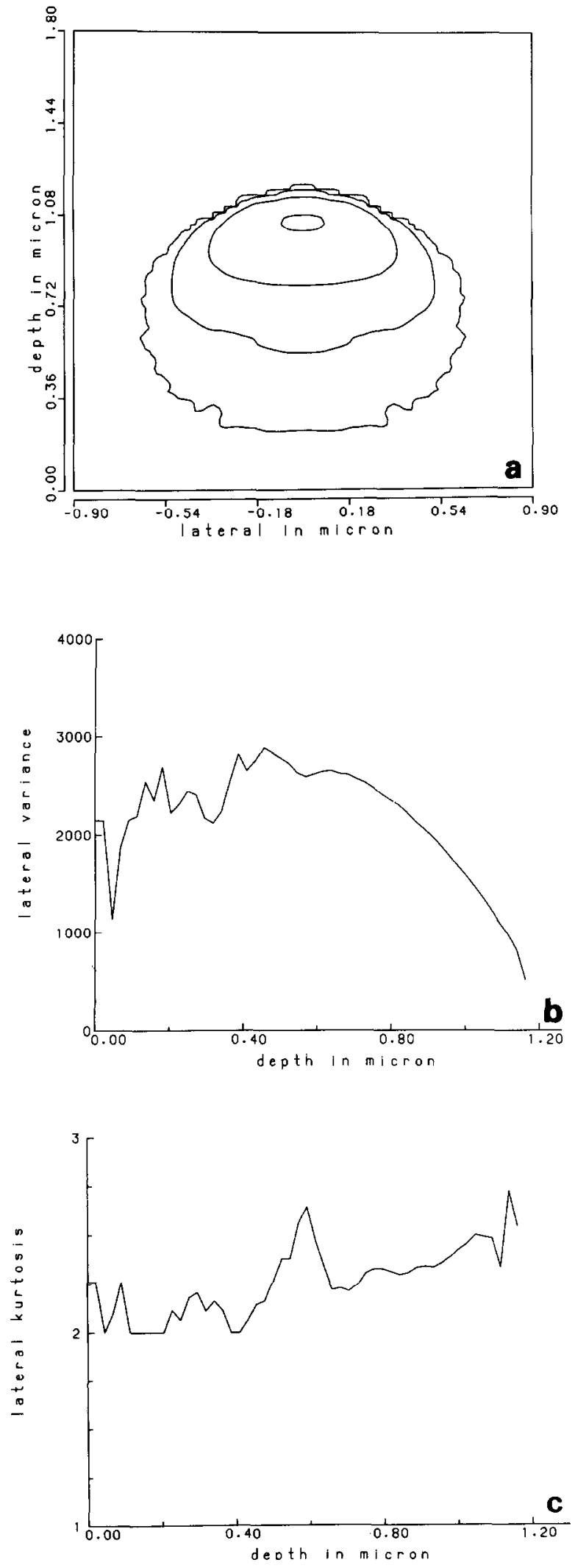

diffusion effect is corrected by subsequent reconstruction cycles. The second part makes sure that the image is a decreasing function with respect to the symmetry axis. It has appeared that the convergence of the iteration process improves by using this filter repeatedly after some (between 5 and 10) iteration cycles.

\subsubsection{Test of the reconstruction algorithm with simulated data}

To test the robustness of the reconstruction algorithm some simulated "measurement" profiles are used. These simulated profiles are obtained by a fit of the Monte Carlo result of a $400 \mathrm{keV}$ boron implant and applying the transformation given by eq. (6). The angles which are used vary from 0 to $80^{\circ}$ in steps of $10^{\circ}$. Because the 2D distribution function is symmetric the negative angles are not necessary.

The results of the tests are shown in figs. $4 a$ to $4 d$. In fig. $4 a$ the result of the reconstruction process is shown with the perfect data. The dotted lines represent the original and the drawn lines represent the reconstruction result. The reconstruction parameters are $80 \times 80$ pixels, $\lambda=0.1, \epsilon=0.01$ (1\% accuracy), 50 iteration cycles, and after every 6 iteration cycles one filter cycle. It can be seen that at depths less then of $0.4 \mu \mathrm{m}$ the reconstruction starts to deviate from the original. This is due to the fact that at large angles those parts of the $2 \mathrm{D}$ distribution fall in the shaded area of fig. $2 b$, i.e. outside the target. In figs. $4 \mathrm{~b}$ to $4 \mathrm{~d}$ the results are shown with corrupted data.

Fig. $4 \mathrm{~b}$ shows the result when the dose of the profiles under different angles is varied randomly by $15 \%$. For this case the effect is largest at depths less than $0.4 \mu \mathrm{m}$. The overall picture at depths greater then $0.4 \mu \mathrm{m}$ is not influenced.

Fig. $4 c$ shows the result when the relative depth scale of the different angles is varied randomly by $5 \%$. It can be seen that now the whole picture is somewhat distorted, but that the overall picture is still quite good.

Fig. 4d shows the result when the profiles of the different angles are shifted randomly in depth by $5 \%$ of the mean projected range. The effect is quite dramatic now.

The conclusion is that the relative position of the profiles of different angles should have an inaccuracy below $1 \%$ to obtain acceptable results. Secondly the result at depths less then $0.4 \mu \mathrm{m}$ is most sensitive to corrupted data.
Fig. 5. Monte Carlo simulation results. a) The 2D distribution function. b) The lateral straggling as a function of the depth. c) The lateral kurtosis as a function of the depth. 


\subsection{A nonlinear optimization technique (NOT)}

The reconstruction algorithm in the former section used no a priori information of the image to be reconstructed. In this section a method is described which uses as much as possible a priori knowledge of the image to be reconstructed. In ref. [5] it is shown that the Line Source Response (LSR) of the ion implantation can be fitted accurately with a function of the form

$\hat{C}_{2 \mathrm{D}}(y, x)=f_{\text {dep }}(y) f_{\text {lat }}(y, x)$.

This function determines the LSR given a few parameters (say 5 or 10). Now we can write

$e=\sum_{i=1}^{m}\left(p_{i}-\mathbb{F}_{i} \hat{C}\right)^{2}$

where

$\hat{C}$ is the fitted function, with parameter vector $a$.

$\mathbb{F}_{i}$ is the $i$ th line integral (6) at a certain depth under a certain angle.

$p_{i}$ is the corresponding measured value.

$e$ is an error estimate to be minimized.

The choice of the fit function is rather ad hoc and depends on the implantation atom and energy. The disadvantage of this method is that it can only be used to calibrate the theoretical image. If any large discrepancy between theory and experiment exists no conclusions can be drawn about the experimental image. In the following section the results of the Monte Carlo simulation are shown, which is used as the test case for the $2 \mathrm{D}$ profiling method.

\section{Results of a Monte Carlo simulation of the test case}

In order to test the reconstruction algorithms, a simulation of a $400 \mathrm{keV}$ boron implant is performed. In this section the simulation result is analyzed and the fit function is defined.

The simulation is performed with a Monte Carlo program based on the simulation program TRIM $[8,9]$. A total number of $10^{5}$ ion trajectories are calculated. The resulting Line Source Response is shown in fig. 5a, where contour lines are drawn at levels 0.9, 0.09, 0.009 and 0.0009 below the top of the $2 \mathrm{D}$ distribution function. The resulting $2 \mathrm{D}$ profile is described by a set of moments. In depth these moments are

$$
\begin{aligned}
& \mu_{\mathrm{dep}}=\int_{-\infty}^{\infty} \int_{-\infty}^{\infty} y C_{2 \mathrm{D}}(y, x) \mathrm{d} x \mathrm{~d} y, \\
& \sigma_{\mathrm{dep}}^{2}=\int_{-\infty}^{\infty} \int_{-\infty}^{\infty}\left(y-\mu_{\mathrm{dep}}\right)^{2} C_{2 \mathrm{D}}(y, x) \mathrm{d} x \mathrm{~d} y, \\
& \gamma_{\mathrm{dep}}=\int_{-\infty}^{\infty} \int_{-\infty}^{\infty}\left(y-\mu_{\mathrm{dep}}\right)^{3} C_{2 \mathrm{D}}(y, x) \mathrm{d} x \mathrm{~d} y / \sigma_{\mathrm{dep}}^{3}, \\
& \beta_{\mathrm{dep}}=\int_{-\infty}^{\infty} \int_{-\infty}^{\infty}\left(y-\mu_{\mathrm{dep}}\right)^{4} C_{2 \mathrm{D}}(y, x) \mathrm{d} x \mathrm{~d} y / \sigma_{\mathrm{dep}}^{4}
\end{aligned}
$$

Note that these are the moments of a standard implantation profile with angle 0 . Now the lateral straggling $o(y)$ and lateral kurtosis $\beta(y)$ as function of the depth $y$ are defined as follows

$$
\begin{aligned}
& \sigma(y)^{2}=\int_{-\infty}^{\infty} x^{2} C_{2 \mathrm{D}}(y, x) \mathrm{d} x \\
& \beta(y)=\int_{-\infty}^{\infty} x^{4} C_{2 \mathrm{D}}(y, x) \mathrm{d} x / \sigma(y)^{4} .
\end{aligned}
$$

These values are obtained by numerical integration of the discretized 2D function. The lateral skewness or other odd moments are zero due to symmetry. In fig. $5 \mathrm{~b}$ and fig. $5 \mathrm{c} \sigma(y)$ and $\beta(y)$ are shown. It can clearly be seen that both the lateral straggle and the lateral kurtosis are depth dependent. Note that when eq. (1) is appropriate, this depth dependency does not occur.

To fit $C_{2 \mathrm{D}}(y, x)$ an approach as in ref. [5] is adopted. The function description is as follows. Eq. (2) is used as starting point

$f(y, x)=f_{\text {dep }}(y) f_{\text {lat }}(y, x)$.

For $f_{\mathrm{dep}}(y)$ a Pearson IV distribution is used, see [10] for a detailed description of the relation between moments and the Pearson parameters. This distribution is the usual projected range-profile (no beveling of the target) and is described by three parameters $\mu_{\mathrm{d}}, \sigma_{\mathrm{d}}$ and $\gamma_{\mathrm{d}}$. For the kurtosis $\beta_{\mathrm{d}}=3 \beta_{\min }$ is chosen. It has appeared that the calculation of the Pearson parameters out of the moments obtained with the numerical integration, do not give the best fit. It appeared that a fit optimization gives better results. Therefore when depth moments are presented both the numerical integrated values and the optimization values are given. For $f_{\text {lat }}(y, x)$ a pseudo Gaussian is chosen

$f_{\text {lat }}(y, x)=a(y) \exp \left(-|b(y) x|^{p(y)}\right)$,

where the depth dependent parameters $a(y), b(y)$ and $p(y)$ are related to the lateral moments as

$$
\begin{aligned}
& 1=\int_{-\infty}^{\infty} f_{\text {lat }}(y, x) \mathrm{d} x, \\
& \sigma(y)^{2}=\int_{-\infty}^{\infty} x^{2} f_{\text {lat }}(y, x) \mathrm{d} x, \\
& \beta(y)=\int_{-\infty}^{\infty} x^{4} f_{\text {lat }}(y, x) \mathrm{d} x / \sigma(y)^{4} .
\end{aligned}
$$

In ref. [5] this relation is derived.

Finally the $\sigma(y)$ and $\beta(y)$ have to be fitted for use of the NOT technique described in section 3.2. For this purpose a piecewise linear function is used

$$
\sigma(y)=\sigma_{1}^{\sigma_{1}}+\frac{\left(y-y_{1}\right)\left(\sigma_{2}-\sigma_{1}\right)}{\left(y_{2}-y_{1}\right)}, \quad \begin{aligned}
& y \leq y_{1}, \\
& \sigma_{2}<y<y_{2}, \\
& y \geq y_{2},
\end{aligned}
$$




$$
\beta(y)=\begin{array}{ll}
\beta_{1} \\
\beta_{1}
\end{array}+\frac{\left(y-y_{1}\right)\left(\beta_{2}-\beta\right)}{\left(y_{2}-y_{1}\right)}, \quad \begin{aligned}
& y \leq y_{1}, \\
& \beta_{2}<y<y_{2}, \\
& y \geq y_{2} .
\end{aligned}
$$

\section{Experimental setup}

The reconstruction algorithm was tested for a boron $\left(B^{11}\right)$ implant with $400 \mathrm{keV}$ acceleration voltage and a dose of $2 \times 10^{15}$. The implantations were performed into amorphous targets of silicon at angles 0 to $80^{\circ}$ in steps of $10^{\circ}$. These amorphous targets were obtained by a low temperature polysilicon deposition on a oxidized silicon wafer. The implants were not in crystalline silicon because channeling under different bevel angles is likely to render the results inconsistent. For each angle one implantation was performed. Because the $2 \mathrm{D}$ distribution function is symmetric the implants at negative angles are not needed. For this experiment it is very important that the relative depth measurements, of the implants at different angles, are very accurate. In a routine SIMS analysis the accuracy of the depth scale will be 5\%.

Numerical experiments with simulated profiles showed that this is insufficient for successful reconstructions. Therefore for each substrate a reference implantation with the boron isotope ${ }^{10} \mathrm{~B}$ was performed under an angle of $0^{\circ}$ with the acceleration voltage 400 $\mathrm{keV}$ and dose $10^{14}$. This reference implant was used to calibrate the relative positions of the ${ }^{11} B$ implants, because for each substrate the ${ }^{10} \mathrm{~B}$ profiles should be identical. In this way the relative positions of the ${ }^{11} B$ implants are within 1\%. Furthermore the reference implant could be used to calibrate the measured dose of the ${ }^{11} \mathrm{~B}$ implants [12], which is especially useful for those angles were reflections are expected to be significant.

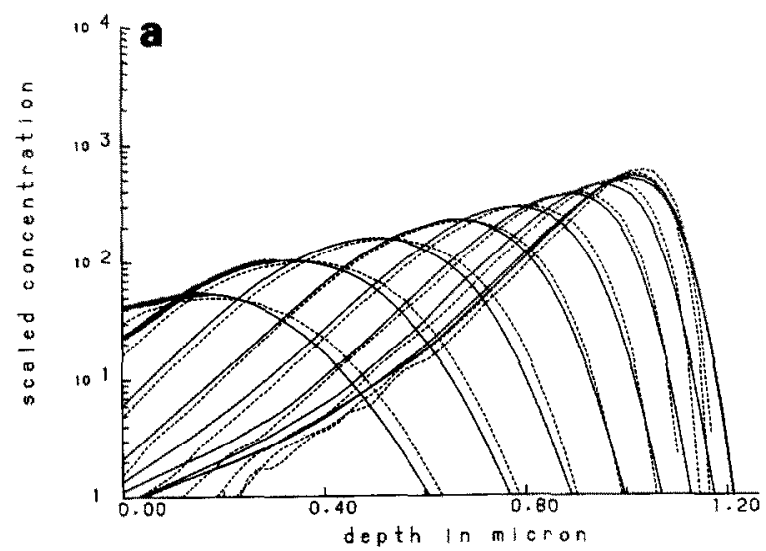

Table 1

Normalized effective dose for the implants for the experimental ones and the ones obtained by simulation. As a reference the cosine is also included

\begin{tabular}{clll}
\hline $\begin{array}{l}\text { Angle } \\
\text { (deg.) }\end{array}$ & Simulated & Experimental & Cosine \\
\hline 0 & 1 & 1 & 1 \\
10 & 0.98 & 0.96 & 0.98 \\
20 & 0.94 & 0.89 & 0.94 \\
30 & 0.87 & 0.81 & 0.87 \\
40 & 0.77 & 0.71 & 0.77 \\
50 & 0.64 & 0.61 & 0.64 \\
60 & 0.50 & 0.46 & 0.50 \\
70 & 0.33 & 0.30 & 0.34 \\
80 & 0.15 & 0.11 & 0.17 \\
\hline
\end{tabular}

All the SIMS analyses were performed at the Philips Research Laboratories with a CAMECA-3F instrument. For the analysis a $10 \mathrm{keV} \mathrm{O} \mathrm{O}_{2}^{+}$beam was used, incident at $37^{\circ}$ with respect to the surface normal. The beam current was about $2 \mu \mathrm{A}$. The raster area was $350 \times 350$ $\mu \mathrm{m}^{2}$ and the analysis area had a diameter of $60 \mu \mathrm{m}$, so redeposition effects from the crater edges are minimal. The minimum attainable decay length, i.e. for an infinitely steep "true" profile, of the SIMS analysis under these conditions was about 0.01 to $0.02 \mu \mathrm{m} /$ decade.

The effective doses under different angles should follow the cosine rule. In table 1 the doses of the different implants scaled with the dose of the $0^{\circ} \mathrm{im}$ plant are listed together with the corresponding cosine of the angle. The same is done with the simulated profiles. The simulated profiles were obtained by using the transformation eq. (6) to the Monte Carlo simulated 2D distribution function. The difference between the cosine and the measured quotient for the angle from 0 to $60^{\circ}$ is due to the inaccuracy of the concentration

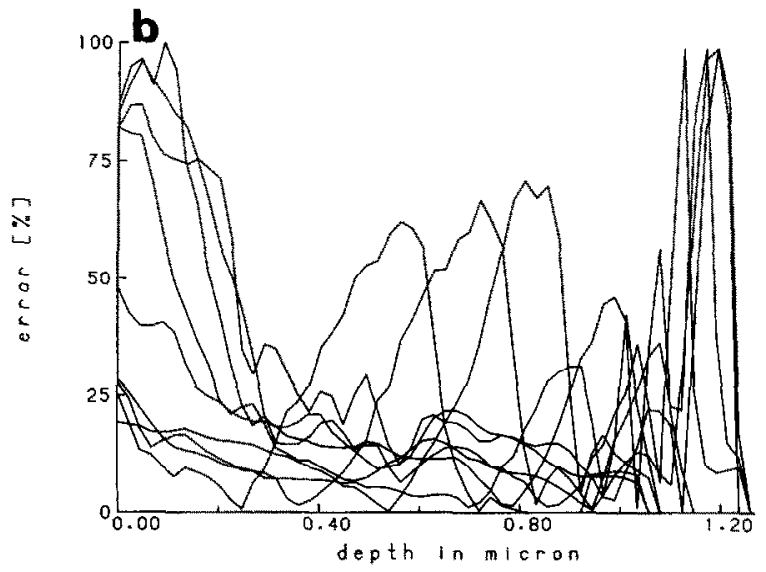

Fig. 6. a) Measured $1 \mathrm{D}$ profiles. The dotted lines are MC results. b) The relative difference between measurement and $\mathrm{MC}$ simulations. 
measurements. The larger difference for the angles $70^{\circ}$ and $80^{\circ}$ is probably due to backscattering and reflections. The quotients of the simulated profiles show a good cosine rule for the angles 0 to $60^{\circ}$. The deviation for $70^{\circ}$ and $80^{\circ}$ is due to backscattering, because a pure reflection is not accounted for in the simulation.

Because the simulated and the measured profiles are otherwise very close it was decided to correct the measured profiles to follow the cosine rule as it is predicted by the simulation. This final measured profiles are shown in fig. 6a together with the Monte Carlo predicted profiles. These plots are scaled to an equivalent dose of $10^{4}$. Furthermore the relative difference between the measurement and the Monte Carlo predictions is plotted as an indication of the appropriateness of the $2 \mathrm{D}$ distribution. It can be seen that the simulated profiles are very close to the measured profiles. In the next two sections the reconstruction to $2 \mathrm{D}$ profiles is discussed.

\section{Discussion of the reconstruction process}

\subsection{Reconstruction with the algebraic reconstruction tech- nique $A R T$}

The output of the $A R T$ algorithm after handling the measured data is shown in fig. 7. In this figure the $2 \mathrm{D}$ distribution function is shown together with the corresponding profiles and the relative errors of these profiles with the measured profiles. It can be seen that some inconsistency in the data exists. This inconsistency appeared to be due to the profiles at $70^{\circ}$ and $80^{\circ}$ near the surface of the substrate. The parts of these profiles are marked by thicker lines in fig. 6. If these thick marked parts are ignored in the reconstruction process the results become much better.

These final results are shown in figs. $8 \mathrm{a}$ to $8 \mathrm{~d}$. Fig. 8 contains the 2D distribution function together with the Monte Carlo result (dotted lines). Also the corresponding profiles and errors compared with the measured profiles are shown. Finally it contains the lateral standard deviation and lateral kurtosis as a function of depth.

\subsection{Reconstruction by optimization with a parameterized function}

All the experimental data is used for the optimization, including the inconsistent thick marked parts of fig. 6. It appeared that the functional description does not allow the inconsistency to exist in the $2 \mathrm{D}$ distribution function. First the profile of the $0^{\circ}$ implant is used to extract the depth parameters $\mu_{\text {dep }} \sigma_{\text {dep }} \gamma_{\text {dep }}$. The kurtosis in the fit is fixed to 1.5 times the minimal allowed kurtosis. Finally the data from $10^{\circ}$ to $80^{\circ}$ is used to extract the lateral parameters, where the in
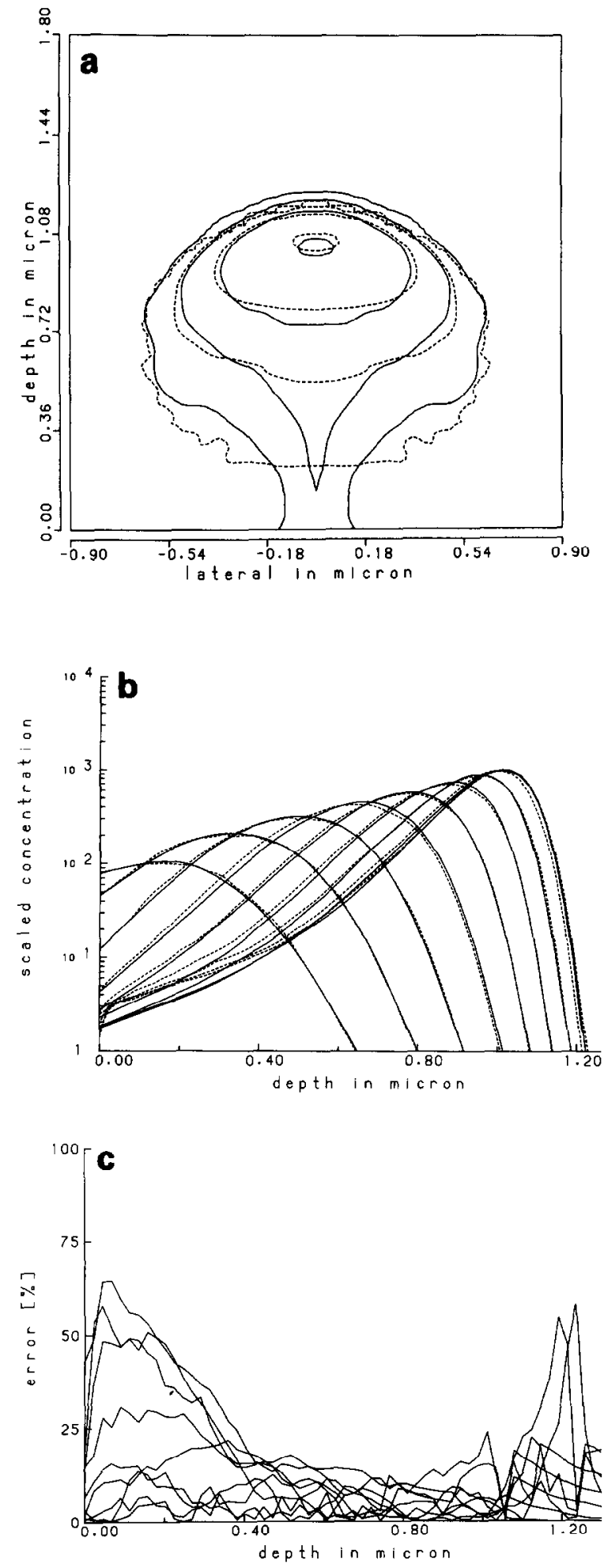

Fig. 7. ART results using all data. a) The $2 D$ distribution function. b) Profiles. Dotted lines result from the reconstructed function. c) Relative difference between the measurement and the reconstruction results. 

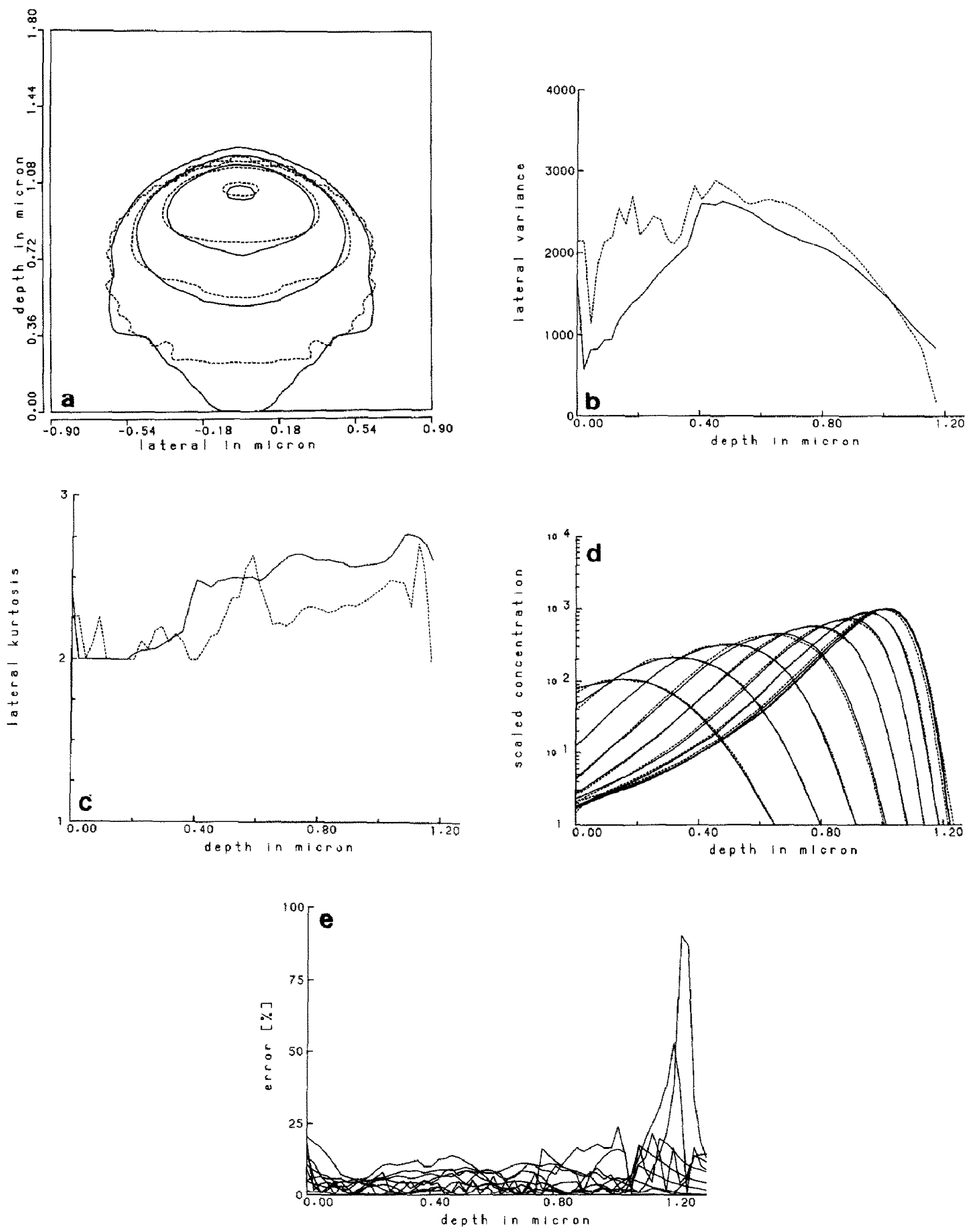

Fig. 8. ART results ignoring thick marked data of fig. 6. a) The 2D distribution function. b) Lateral straggling as a function of the depth. c) Lateral kurtosis as a function of the depth. d) Profiles. Dotted lines result from the reconstructed function. e) Relative difference between the measurement and the reconstruction results. 

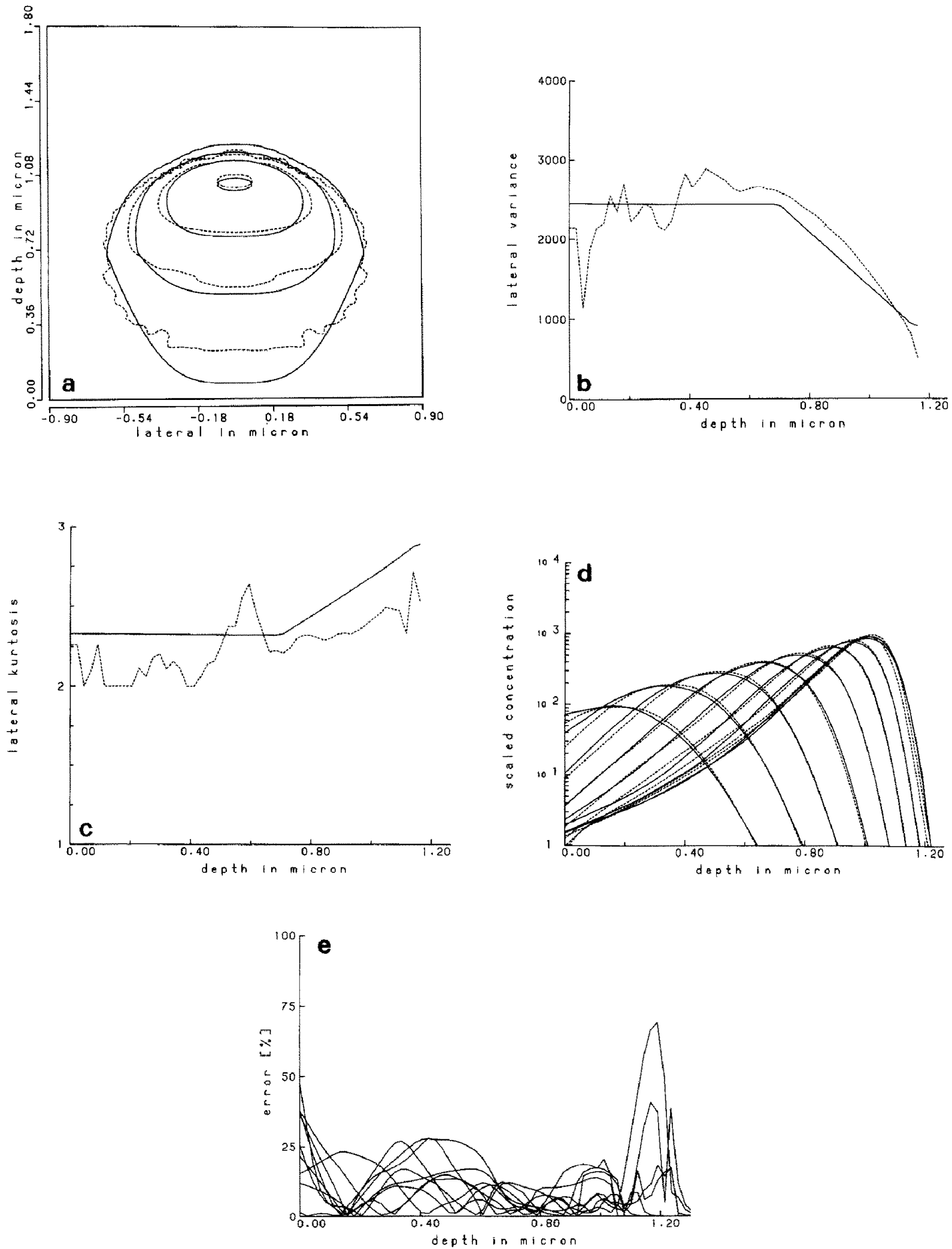

Fig. 9. NOP results using all data. (a) to (e) as for fig. 8. 
Table 2

Extracted parameters for the optimization technique. For the depth moments the values obtained by numerical integration are given between brackets

\begin{tabular}{ccccccccc}
\hline$\mu_{\text {dep }}$ & $\sigma_{\text {dep }}$ & $\gamma_{\text {dep }}$ & $y_{1}$ & $y_{2}$ & $\sigma_{1}$ & $\sigma_{2}$ & $\beta_{1}$ & $\beta_{2}$ \\
\hline 0.96 & 0.13 & -3.9 & 0.6 & 1.2 & 0.28 & 0.07 & 2.25 & 2.59 \\
$(0.97)$ & $(0.10)$ & $(-1.2)$ & & & & & & \\
\hline
\end{tabular}

depth parameters were fixed to the previously obtained values. As starting values for the parameters some values were used which were close to the ART reconstruction results. In fig. 9 the results are shown of the reconstruction analog to the ART results. In table 2 the extracted parameters are shown.

\subsection{Discussion}

The measured profiles appear to contain some inconsistent parts. First at large tilt angles back scattering effects give some distortion of the data. The ART technique is very sensitive to this distortion. If the inconsistent parts are removed from the reconstruction data. The technique works, however, quite well. These inconsistent parts are thick marked in fig. 6 . The NOT method is not sensitive to this inconsistency. The results are identical with or without the inconsistent data because the functional description does not allow for a distortion and the error term is mainly determined by other parts in the data.

Second both methods appear to have difficulties fitting the profiles at $0^{\circ}$ and $10^{\circ}$ at the edge of the profiles at depths greater than $1.2 \mu \mathrm{m}$. The error indicator however is very sensitive for minor distortions in the depth scale at these edges, so any error is exaggerated. The measured profiles at 0 and $10^{\circ}$ are almost identi$\mathrm{cal}$, whereas according to the simulations some difference should exist. This difference is however within the $1 \%$ inaccuracy of the depth scale. In the case that the measured profile at 0 is expanded in depth with $0.5 \%$ and the measured profile at $10^{\circ}$ is shortened with $0.5 \%$, the error indicators reduce significant but, the reconstruction result is not significantly influenced. The depth resolution of the SIMS analysis is not expected to disturb the reconstruction. The maximum decay in the experimental data is about $0.04 \mu \mathrm{m} /$ decade attained for $0^{\circ}$, whereas the decay length caused by the SIMS analyses conditions is about 0.01 to $0.02 \mu \mathrm{m} /$ decade.

Both reconstruction techniques give about the same results. The ART technique however can reveal more details of the actual shape of the $2 \mathrm{D}$ distribution. The NOT technique will always be limited by the appropriateness of the functional description. A possible measure of the reconstruction quality is the average value of the error estimator for all the measured data. For the ART technique this value is about $5 \%$ and for the NOT technique this value is about $15 \%$. The NOT results can possibly be improved by using a better piecewise approximation to the moments as a function of the depth [13]. The surface truncation of the profiles at large tilt angles causes the ART technique to give inaccurate results at depths less than $0.4 \mu \mathrm{m}$, as was demonstrated in section 3 . The quality of the NOT technique in that region is determined by the appropriateness of the functional description. For the used fit function [eqs. (19) and (20)] the fit with the Monte Carlo data is not accounted for in this region, so for both methods the results at depths less than $0.4 \mu \mathrm{m}$ should be considered not significant.

The Monte Carlo simulation results are close to the measured results. However both reconstruction techniques indicate some deviations between the simulation and the measurement. First the lateral standard devia* tion near the peak of the measured 2D distribution function is not as small as indicated by the simulation. The measured $2 \mathrm{D}$ distribution function is not as steep at the back side as the simulated one. Finally the lateral kurtosis of the measured 2D distribution function is a bit larger. i.e. more near to a Gaussian form (kurtosis = 3).

The fact that the ART reconstruction process is successful implies that the assumptions made to derive eq. (6) are correct. To stress the significance of this fact these assumptions are given as: a) the implantation process in a target may be expressed as a convolution of one Line Source Response. b) the properties of the $2 \mathrm{D}$ distribution are independent of the implantation angle.

\section{Conclusions}

It has been shown that it is possible to measure a two dimensional ion distribution resulting from implantation into amorphous targets using a line source. The measurement methods combine SIMS depth profiling methods with inverse Radon transforms. The depth profiles are obtained from implantations into beveled targets. The measured distributions for $400 \mathrm{keV}^{11} \mathrm{~B}$ ions implanted into amorphous silicon were found to be closely similar to theoretical distributions obtained using the TRIM computer code. The accuracy of the depth scales of the profiles appeared to be very important for successful inverse transforms. By using ${ }^{10} \mathrm{~B}$ implants 
incident at $0^{\circ}$ as a reference profile, an inaccuracy in the depth of $1 \%$ has been obtained.

We wish to thank Tom Aarnink of our institute for processing all the wafers. Desiree Delang from PRL for her helpful discussions and finally Jan Zandhuis for implementing many of the reconstruction ideas.

\section{References}

[1] S. Furukawa, H. Matsumara and H. Ishiware, Jpn. J. Appl. Phys. 11 (2) (1972) 134.

[2] S. Furukawa and H. Matsumara, Appl. Phys. Lett., 22 (3) (1973) 97.

[3] G. Fuse, H. Umimoto, S. Odanaka, M. Wadabayashi, M. Fukumoto and T. Ohzone, J. Electrochem. Soc. 133 (5) (1986) 996.

[4] H. Okabayashi and D. Shinoda, J. Appl. Phys. 44 (19) (1973) 4220.
[5] G. Hobler, E. Langer and S. Sclberherr, Solid State Elcctron. 30 (4) (1987) 445.

[6] R.M. Lewitt, Proc. IEEE 71 (3) (1983) 389.

[7] Y. Censor, Proc. IEEE 71 (3) (1983) 409.

[8] J.F. Ziegler, J.P. Biersack and U. Littmark, (eds.) The Stopping and Range of Ions in Solids, vol. 1 of the Stopping and Ranges of Ions in Matter, (Pergamon Press, New York, 1985).

[9] E. van Schie and J. Middelhoek, Two methods to improve the performance of Monte Carlo Simulations of Ion Implantation in Amorphous Targets, accepted for publication in IEEE Trans. on Computer Aided Design.

[10] S. Selberherr, Analysis and Simulation of Semiconductor Devices, (Springer, Wien, New-York, 1984) pp. 47-60.

[11] P.B.M. Wolbert, PROMEA: a Flexible Parameter Extractor, MSc Thesis University of Twente (1986).

[12] P.C. Zalm et al., to be published.

[13] R. Oven, D.G. Ashworth and C. Hill, Proc. 3rd Int. Conf. on Simulation of Semiconductor Devices and Processes, University of Bologna (Sept. 1988). 